\title{
Modulation of rat pulmonary carcinogen-metabolising enzyme systems by the isothiocyanates erucin and sulforaphane
}

\author{
Natalya Hanlon, *Nick Coldham, *Maurice J Sauer and Costas Ioannides
}

Centre of Toxicology, Faculty of Health and Medical Sciences, University of Surrey,

Guildford, Surrey, GU2 7XH, and *TSE Molecular Pathogenesis and Genetics

Department, Veterinary Laboratories Agency Weybridge, Woodham Lane, New Haw, Addlestone, Surrey, KT15 3NB, UK

Corresponding author: Professor C Ioannides

Centre of Toxicology

Faculty of Health and Medical Sciences

University of Surrey

Guildford, Surrey

GU2 7XH, UK

Tel. No: +441483689709

Fax No: +44 1483686401

E-mail: c.ioannides@surrey.ac.uk 


\section{Abstract}

The objective of this study was to evaluate the potential of the structurally-related aliphatic isothiocyanates erucin and sulforaphane to modulate the pulmonary carcinogen-metabolising enzyme systems in rat lung, a target organ of their chemopreventive activity. Precision-cut rat lung slices were prepared and incubated for 24 hours with a range of concentrations of either erucin or sulforaphane, up to 50 $\mu \mathrm{M}$. Neither compound modulated the $O$-deethylation of ethoxyresorufin whereas they elevated markedly CYP1A1 and, to a lesser extent, CYP1B1 apoprotein levels. Neither compound influenced the $O$-depentylation of pentoxyresorufin or CYP2B apoprotein levels, but sulforaphane caused a modest increase in CYP3A2 apoprotein levels. Pulmonary quinone reductase activity, monitored using 3-(4,-5dimethylthiazo-2-yl)-2,5-diphenyltetrazolium bromide as substrate, was markedly upregulated by both compounds and was paralleled by a similar rise in protein levels. Both compounds increased cytosolic glutathione $S$-transferase activity, measured using 1-chloro-2,4-dinitrobenzene as the accepting substrate; a modest rise was seen in GST $\alpha$ protein levels, determined immunologically, whereas GST $\pi$ levels were unaffected by the same treatment. Finally, both erucin and sulforaphane increased total glutathione concentration in lung cytosol. It is concluded that these aliphatic isothiocyanates have the potential to antagonise the carcinogenicity of pulmonary carcinogens by stimulating the in situ detoxication of their DNA-binding genotoxic metabolites.

Key words: erucin; sulforaphane; isothiocyanates; CYP1; chemoprevention; quinone reductase 


\section{Introduction}

Numerous epidemiological studies have established that dietary habits play a pivotal role in the aetiology and progression of human diseases including cancer [1]. A large number of dietary phytochemicals have been recognised with potential chemopreventive activity, and in laboratory studies they could antagonise the carcinogenicity of chemicals in animal models. One of the most extensively studied classes of chemopreventive phytochemicals is the isothiocyanates. These are encountered in cruciferous vegetables where they occur in the form of glucosinolates but are released following exposure to the enzyme myrosinase ( $\beta$-thioglucoside glucohydrolase) that comes into contact with these compounds during the harvesting, chopping and mastication of these vegetables [2] Moreover, glucosinolates that progress intact to the human intestine intact may be metabolised by microbial myrosinases, further contributing to the release of isothiocyanates [3].

Several studies reported that high intake of isothiocyanates in the diet was associated with a reduced risk of lung cancer in humans $[4,5]$. In laboratory studies, a major target organ of isothiocyanates is the lung, where many isothiocyanates suppressed tumourigenesis in mouse and rat induced by exposure to pulmonary carcinogens. For example, in $\mathrm{A} / \mathrm{J}$ mice, the aromatic isothiocyanates benzyl and phenethyl isothiocyanate decreased the tumour yield in animals exposed to the carcinogens benzo[a]pyrene and 4-(methylnitrosamino)-1-(3-pyridyl)-1-butanone (NNK) [6]. In the same mouse strain, benzyl isothiocyanate was an effective inhibitor of lung tumourigenesis induced by 5-methylchrysene, dibenz[a,h]anthracene, benzo[a]pyrene and diethylnitrosamine $[7,8]$. Phenethyl isothiocyanate anatagonised the carcinogenicity of the tobacco carcinogen NNK and inhibited DNA-adduct formation in rat [9]. Finally, the malignant progression of lung adenomas induced by 
a mixture of benzo[a]pyrene and NNK was inhibited by sulforaphane (1isothiocyanato-4-(methylsulphinyl) butane) and phenethyl isothiocyanate [10].

A major mechanism through which isothiocyanates exert their chemopreventive effect is by modulating the enzymes involved in the metabolism of chemical carcinogens, altering the balance of activation/deactivation so as to favour the latter. Isothiocyanates are believed to limit the production of DNA-binding electrophilic metabolites by inhibiting their cytochrome P450-mediated generation on one hand, and stimulating their detoxication on the other. Indeed, a number of isothiocyanates have been shown to influence hepatic cytochrome P450 expression as well as phase II enzyme systems such as quinone reductase and glutathione $S$ transferase [11]. In contrast to the liver, very few studies have been undertaken in the lung, despite the fact that this a major target organ for the chemopreventive activity of isothiocyanates. Moreover, the limited studies carried out have identified tissue differences in the response of lung and liver to isothiocyanates. Phenethyl isothiocyanate up-regulated markedly quinone reductase in the liver but not in the lung of rats $[12,13]$.

Consequently, we investigated the potential of sulforaphane and erucin (1isothiocyanato-4-(methylthio)-butane), a structurally related isothiocyanate present in rocket salad, to modulate carcinogen-metabolising enzyme systems in rat lung; it has already been documented that these isothiocyanates modulate these enzyme systems in rat liver [14]. The studies reported here were conducted in vitro utilising precisioncut lung slices [15] The principal advantages of this in vitro system are that it maintains tissue architecture and cell-cell communication, and allows the facile use of a wide range of concentrations of chemicals, that would otherwise necessitate large animal numbers for commensurate in vivo studies [16]. It is particularly suited to the 
lung, a heterogeneous tissue composed of many different cell types that differ markedly in their carcinogen-metabolising enzyme profile [17]. Studies emanating from our laboratory have established that precision-cut slices are an appropriate system for use in evaluating the potential of xenobiotics to modulate cytochrome P450 and phase II activities in the lung [18].

\section{Materials and methods}

\section{Materials}

Sulforaphane and erucin (LKT Laboratories, Minnesota, USA), NADPH, ethoxyresorufin, pentoxyresorufin, resorufin, 1-chloro-2,4-dinitrobenzene (CDNB), glutathione reductase, peroxidase-linked anti-rabbit and anti-goat antibodies raised in rabbits (Sigma Co. Ltd., Poole, Dorset, UK) were all purchased. Rat anti-CYP1A1, recognising both CYP1A1 and CYP1A2, anti-CYP1B1, anti-CYP2B and antiCYP3A2 antibodies were obtained from BD Biochemicals (Oxford, UK); antibodies to human GST P1-1, A1-1 and M1-1 (Calbiochem, Merck, UK) and antibody to human quinone reductase (abcam Cambridge, UK) were similarly purchased.

\section{Preparation and incubation of slices}

Male Wistar albino rats (about 180g) were obtained from B\&K Universal Ltd (Hull, East Yorkshire, UK). The animals were housed at $22 \pm 2{ }^{\circ} \mathrm{C}, 30-40 \%$ relative humidity, in an alternating 12-hr light:dark cycle with light onset at $07.00 \mathrm{hr}$. Precision-cut rat lung slices were prepared as previously described [15]. Briefly, lungs were perfused intratracheally with agarose $(0.75 \% \mathrm{v} / \mathrm{w})$ at $37^{\circ} \mathrm{C}$. Agar was allowed to solidify, and lung slices $(600 \mu \mathrm{m})$ were prepared from cylindrical cores $(8 \mathrm{~mm})$ using a Krumdieck tissue slicer (Alabama Research and Development Corporation, 
Munsford, AL, USA). The multiwell plate procedure, using 12-well culture plates, was used to culture the slices. The culture medium was essentially that described by Lake et al [19], and one slice was placed in each well, in $1.5 \mathrm{ml}$ of culture medium. Slices were initially pre-incubated for $60 \mathrm{~min}$ in order to slough off any dead cells due to slicing, before being transferred to fresh medium and incubated for a period of 24 hours in the presence of a range of concentrations of sulforaphane or erucin on a reciprocating plate shaker housed in a humidified incubator, at a temperature of $37{ }^{\circ} \mathrm{C}$ and under an atmosphere of $95 \% \operatorname{air} / 5 \% \mathrm{CO}_{2}$.

\section{Enzyme assays}

At the end of incubation, slices were removed from the medium, homogenised and post-mitochondrial fractions were prepared by differential centrifugation and stored at $-80^{\circ} \mathrm{C}$ until use. When required, samples were thawed and resolved to microsomal and cytosolic fractions by centrifugation (105 000g x 1 hour). The dealkylations of ethoxy- [20] and pentoxyresorufin [21] were determined using the microsomal fraction. The following assays were performed using the cytosol; quinone reductase using MTT [3-(4,-5-dimethylthiazo-2-yl)-2,5-diphenyltetrazolium bromide] as substrate [22], glutathione $S$-transferase activity using CDNB as accepting substrate [23], and total glutathione [24]. Protein concentration was determined in both fractions using bovine serum albumin as standard [25]. Finally, in order to determine changes in enzyme protein expression, pooled pulmonary microsomal and cytosolic proteins were resolved by electrophoresis and incubated with the primary antibody and the corresponding peroxidase-linked secondary antibody. Molecular markers were run concurrently in all cases. Immunoblots were quantitated by densitometry using the GeneTool software (Syngene Corporation, Cambridge, UK). 
Lactate dehydrogenase (LDH) release from the lung slices into the incubation medium was used as an index of cytotoxicity. The LDH concentration was measured employing a cytotoxicity detection kit plus (Roche Diagnostics, Mannheim, Germany), using three slices per concentration of isothiocyanate. On completion of a 24-h incubation, the culture medium was aspirated and the tissue slices were each homogenised in $1.5 \mathrm{ml}$ of phosphate buffered saline (PBS), $\mathrm{pH}$ 7.4. The media and homogenates were centrifuged at $2000 \mathrm{x}$ g for $5 \mathrm{~min}$ at $4^{\circ} \mathrm{C}$ using a bench centrifuge. Duplicate aliquots $(0.1 \mathrm{ml})$ were used for $\mathrm{LDH}$ analysis according to the manufacturer's instructions.

\section{Statistical evaluation}

Statistical evaluation was carried out using the Student's t-test.

\section{Results}

Neither erucin (Figure 1) nor sulforaphane (Figure 2) influenced the $O$-dealkylations of ethoxy- and pentoxyresorufin in rat lung slices, except for a decrease that occurred only at the highest concentrations. Immunological determination of apoprotein levels indicated a concentration-dependent increase in CYP1A1 apoprotein levels brought about by both isothiocyanates, which diminished at the highest concentrations (Figure 3). A similar, but less pronounced, rise was also observed in CYP1B1 apoprotein levels following exposure of lung slices to these isothiocyanates. A modest rise in CYP3A2 proteins was also noted in the case of sulforaphane but not erucin; neither isothiocyanate influenced CYP2B apoprotein levels.

Erucin and sulforaphane elevated, in a concentration-dependent manner, quinone reductase activity when incubated with lung slices for 24 hours; both 
compounds also brought about a rise in glutathione $S$-transferase activity (Figures 1 and 2). Similarly, both compounds elevated total glutathione levels with sulforaphane being more effective. Quinone reductase protein levels were trebled by treatment of lung slices with both isothiocyanates (Figure 4). Finally, the isothiocyanates moderately elevated GST $\alpha$ protein levels and slightly GST $\mu$ levels, with no change in GST $\pi$ (Figure 4).

Lung slice viability was assessed using as biomarker the secretion of lactate dehydrogenase in to the culture medium. Sulforaphane displayed no toxicity up to a concentration of $10 \mu \mathrm{M}$ but a concentration-dependent increase in lactate dehydrogenase secretion was noted at higher concentrations (Figure 5). Erucin displayed toxicity only at the 50 and $100 \mu \mathrm{M}$ concentrations.

\section{Discussion}

Lung is a major target tissue for many chemical carcinogens of human relevance, including polycyclic aromatic hydrocarbons, heterocyclic amines and nitrosamines, all of which are present in tobacco, albeit at different levels [26]. These carcinogens are indirect-acting so that in order to elicit their carcinogenicity they must first be metabolically converted to DNA-binding species, and this process is largely catalysed by cytochromes P450 [27]. Such bioactivation can take place in situ, but there is also experimental evidence to support the view that reactive intermediates generated in the liver, the principal site of bioactivation, may be transported to extrahepatic tissues although the underlying mechanism(s) remain elusive [28, 29]. A number of studies have linked pulmonary CYP1 activity to human cancer incidence, denoting the importance of in situ metabolism. For example, a very significant correlation has been reported between pulmonary levels of CYP1A1 and DNA adduct levels with the 
tobacco-derived polycyclic aromatic hydrocarbons [30]. Among smokers, those who develop tumours display high CYP1A1 activity in the lung [31]. Moreover, the bioactivation of polycyclic aromatic hydrocarbons by CYP1A1 appears to occur in the same part of the airways and in the same cell types in which peripheral carcinomas are observed [32]. However, the availability of DNA-binding intermediates is not solely dependent on their rate of generation, as these may be detoxified by enzyme systems such as quinone reductase and the glutathione $S$ transferases. Thus, an effective chemopreventive agent would be a chemical that suppresses the cytochrome P450-catalysed formation and/or increases phase II detoxication of the DNA-binding carcinogen metabolites. Consequently, we investigated the ability of erucin and sulforaphane, two naturally-occurring isothiocyanates, to modulate the profile of cytochrome P450 and phase II activities in rat lung.

When incubated with precision-cut rat lung slices, both erucin and sulforaphane modulated cytochrome $\mathrm{P} 450$ expression, but in an isoform-specific manner. Both isothiocyanates led to a marked elevation of CYP1A1 and, to a lesser extent, CYP1B1 apoprotein levels, whereas CYP2B was unaffected and CYP3A2 was moderately up-regulated only by sulforaphane. A similar qualitative picture was obtained in liver slices incubated with these isothiocyanates under identical conditions [14]. Despite the marked rise in CYP1A1 expression, no commensurate increase in activity, as exemplified by the $O$-deethylation of ethoxyresorufin, was observed. We have already established in studies conducted in rat liver microsomal preparations that both erucin and sulforaphane are effective mechanism-based inhibitors of this cytochrome P450 enzyme [33], and most likely the same mechanism is responsible for the lack of increase in CYP1A1 activity in the lung. The metabolites of 
isothiocyanates responsible for this inhibition of activity are believed to be isocyanates that are generated by cytochrome P450-mediated desulphuration [34]. Clearly the lung, despite its much lower concentration of cytochrome P450 compared with the liver, is capable of metabolically converting erucin and sulforaphane to products that react with and inactivate the enzyme.

The effects of these isothiocyanates on carcinogen-metabolising enzymes are not confined to the cytochrome P450 enzyme system as changes were also observed in phase II activities. Both compounds enhanced quinone reductase activity that was paralleled by a rise in protein levels indicating that increased enzyme availability is responsible for the increased activity. As this enzyme system is responsible for the detoxification of quinones, it may be inferred that these isothiocyanates have the potential to antagonise the carcinogenicity of polycyclic aromatic hydrocarbons in the lung through such a mechanism. In addition to quinones, polycyclic aromatic hydrocarbons are bioactivated to primary and dihydrodiol epoxides which are electrophiles that readily interact covalently with DNA [35]. These intermediates may be neutralised by conjugation with glutathione catalysed by the cytosolic glutathione $S$-transferases. Both erucin and sulforaphane stimulated glutathione $S$-transferase activity when incubated with precision-cut lung slices, as previously observed in liver slices [36]. Glutathione $S$-transferase activity was monitored using CDNB, an accepting substrate for a number of the cytosolic transferases [37]. Immunoblot analysis revealed that the effects of these isothiocyanates on the glutathione $S$ transferase system are isoform-specific, in that GST $\alpha$ was clearly elevated with only a marginal rise in GST $\mu$ and no effect in GST $\pi$. A similar induction profile was obtained when liver slices were incubated with erucin and sulforaphane under identical conditions [36]. As observed in liver slices [36], an increase in total 
glutathione levels was manifested by both compounds, this effect is probably of greater importance in the lung where basal levels of glutathione are much lower. An increase in $\gamma$-glutamylcysteine synthetase, the rate-limiting enzyme in glutathione biosynthesis, is believed to be the responsible mechanism [2].

At the highest concentrations of the isothiocyanates $(>25 \mu \mathrm{M})$, the dealkylations of the two alkoxyresorufins dropped below control levels, most likely reflecting decreased viability of the lung slices as exemplified by increased leakage of lactate dehydrogenase. As exemplified by the leakage of lactate dehydrogenase, erucin appears to be less toxic than sulforaphane to rat lung slices. It should be emphasised that such concentrations are unlikely to be achieved following single dietary intake of these isothiocyanates; when rats were treated with a single dose of sulforaphane, simulating human intake, the maximum plasma concentrations achieved were $<0.3 \mu \mathrm{M}[38]$. However, plasma concentrations of isothiocyanates are likely to be higher in individuals consuming cruciferous vegetables daily or take nutritional sepplements of isothioganates. Although this concentration is lower than the lowest employed in the current study $(1 \mu \mathrm{M})$, in animal anticarcinogenicity studies, not only are these isothiocyanates given at much higher doses, but also daily for almost the entire life span of the animals, so that the steady-state state plasma isothiocyanate concentrations in these animals are expected to be orders of magnitude higher. Therefore, the wide range of concentrations we employed in our studies (1-50 $\mu \mathrm{M})$ represents the lowest plasma concentration and the maximum concentration tolerated by the slices. As far as human intake is concerned, a single intake of $100 \mathrm{~g}$ of broccoli, a relatively modest amount, achieved a mean peak concentration of nearly $1 \mu \mathrm{M}$ [39]. However, plasma concentrations of isothiocyanates are likely to be higher in individuals consuming cruciferous vegetables daily or take nutritional supplements of 
isothiocyanates. Finally, it is pertinent to point out that recent work suggests that GST phenotype may be a determining factor in plasma concentrations, since conjugation with glutathione is the primary route of metabolism of isothiocyanates, so that generalisations about plasma levels could be misleading [40].

In conclusion, both erucin and sulforaphane influence the activity of carcinogen-metabolising enzymes in rat lung slices to a similar extent. As erucin is a major metabolite of sulforaphane, at least in rat [41, 42], it is conceivable that it contributes to its chemopreventive effects. No major tissue differences between lung and liver slices $[14,36]$ could be discerned in their response to the two isothiocyanates. The most marked effects manifested by sulforaphane and erucin were enhanced quinone reductase and glutathione $S$-transferase activity, and this mechanism may be largely responsible for the documented chemopreventive effect of isothiocyanates in the lung of animals exposed to polycyclic aromatic hydrocarbons. Finally, the current study further highlights the suitability of the precision-cut tissue system in evaluating the potential of chemicals to modulate these enzyme systems.

\section{Acknowledgements}

The authors acknowledge with gratitude the financial support of the Association for International Cancer Research. 


\section{References}

[1] S.D. Stan, S. Kar, G.D. Stoner, S.V. Singh, Bioactive food components and cancer risk reduction. J. Cell Biochem. 104(2008)339-356.

[2] Y. Zhang, P. Talalay, G.C. Cho, G.H. Posner, A major inducer of anticarcinogenic protective enzymes from broccoli: isolation and elucidation of structure. Proc. Natl. Acad. Sci. 89(1992)2399-2403.

[3] S.M. Getahun, F.L. Chung, Conversion of isothiocyanates in humans after ingestion of cooked watercress, Cancer Epidemiol. Biomark. Prev. 8(1999)447-451.

[4] H. Zhao, H.B. Grossman, L.M. Hernandez, C.P. Dinney, X. Wu, Dietary isothiocyanates, GSTM1, GSTT1, NAT2 polymorphisms and bladder cancer risk. Int. J. Cancer 120(2007)2208-2213.

[5] M.R. Spitz, C.M. Duphorne, M.A. Detry, P.C. Pillow, C.I. Amos, L. Lei, M. de Andrade, X.J. Gu, W.K. Hong, X.F. Wu, Dietary intake of isothiocyanates: Evidence of a joint effect with glutathione S-transferase polymorphisms in lung cancer risk, Cancer Epidemiol. Biomark. Prev. 9(2000)1017-1020.

[6] S.S. Hecht, P.M. Kenney, M. Wang, N. Trushin, P. Upadhyaya, Effects of phenethyl isothiocyanate and benzyl isothiocyanate, individually and in combination, on lung tumorigenesis induced in $\mathrm{A} / \mathrm{J}$ mice by benzo[a]pyrene and 4(methylnitrosamino)-1-(3-pyridyl)-1-butanone, Cancer Lett. 13(2000)49-56.

[7] S.S. Hecht, P.M. Kenney, M. Wang, N. Trushin, P. Upadhyaya, Benzyl isothiocyanate: an effective inhibitor of polycyclic aromatic hydrocarbon tumorigenesis in A/J mouse lung, Cancer Lett. 187(2002)87-94.

[8] L.W. Wattenberg, Inhibitory effects of benzyl isothiocyanate administered shortly before diethylnitrosamine or benzo[a]pyrene on pulmonary and forestomach neoplasia in A/J mice, Carcinogenesis 8(1987)1971-1973. 
[9] M.A. Morse, C.X. Wang, G.D. Stoner, S. Mandal, P.G. Conran, S.G. Amin, S.S. Hecht, F/L. Chung, 1989. Inhibition of 4-(methylnitrosamino)-1-(3-pyridyl)-1butanone-induced DNA adduct formation and tumorigenicity in the lung of F344 rats by dietary phenethyl isothiocyanates, Cancer Res. 49(1989)549-553.

[10] C.C. Conaway, C.-X. Wang, B. Pittman, Y.-M. Yang, J.E. Schwartz, D. Tian, E.J. McIntee, S.S. Hecht, F.-L. Chung, 2005. Phenethyl isothiocyanate and sulforaphane and their $\mathrm{N}$-acetylcysteine conjugates inhibit malignant progression of lung adenomas induced by tobacco carcinogens in $\mathrm{A} / \mathrm{J}$ mice, Cancer Res. 65(2005)8548-8557.

[11] C.C. Conaway, Y.-m. Yang, F.-L. Chung, Isothiocyanates as cancer chemopreventive agents: Their biological activities and metabolism in rodents and humans, Curr. Drug Met. 3(2002)233-255.

[12] Z. Guo, T.J. Smith, E. Wang, N. Sadrieh, Q. Ma, P.E. Thomas, C.S. Yang, Effect of phenethyl isothiocyanate, a carcinogenesis inhibitor, on xenobioticmetabolizing enzymes and nitrosamine metabolism in rats, Carcinogenesis 13(1992)2205-2210.

[13] N. Konsue, C. Ioannides,. Tissue differences in the modulation of rat cytochromes P450 and phase II conjugation systems by dietary doses of phenethyl isothiocyanate, Food Chem. Toxicol. In Press.

[14] N. Hanlon, N. Coldham, M.J. Sauer, C. Ioannides, Up-regulation of the CYP1 family in rat and human liver by the aliphatic isothiocyanates erucin and sulforaphane, Toxicology (2008) In Press.

M. Umachandran, J. Howarth, C. Ioannides, Metabolic and structural viability of precision-cut rat lung slices in culture, Xenobiotica 34(2004)771-780. 
[16] C. Lerche-Langrand, H.J. Toutain, Precision-cut liver slices: characteristics and use for in vitro pharmaco-toxicology. Toxicology 153(2000)221-253.

[17] J. Hukkanen, O. Pelkonen, J. Hakkola, H. Raunio, Expression and regulation of xenobiotic-metabolizing cytochrome P450 (CYP) enzymes in human lung, Crit. Rev. Toxicol. 32(2002)391-411.

[18] D.S. Pushparajah, M. Umachandran, K.E. Plant, N. Plant, C. Ioannides, Evaluation of the precision-cut liver and lung slice systems for the study of induction of CYP1, epoxide hydrolase and glutathione $S$-transferase activities, Toxicology 231(2007)68-80.

[19] B.G. Lake, J.A. Beamand, A.C. Japenga, A. Renwick, S. Davies, R.J. Price, Induction of cytochrome P-450-dependent enzyme activities in cultured rat slices. Food Chem. Toxicol. 31(1993)377-386.

[20] M.D. Burke, R.T Mayer, Ethoxyresorufin: direct fluorimetric assay of a microsomal O-dealkylation which is preferentially inducible by 3methylcholanthrene, Drug Metab. Disp. 2(1974)583-588.

[21] R.A. Lubet, R.T Mayer, J.W. Cameron, R.N. Nims, M.D. Burke, T.Wolf, F.P. Guengerich, Dealkylation of pentoxyresorufin: a rapid and sensitive assay for measuring induction of cytochrome(s) P450 by phenobarbital and other xenobiotics in the rat, Arch. Biochem. Biophys. 238(1985)43-48.

[22] H.J. Prohaska, A.B. Santamaria, Direct measurement of NAD(P)H:quinone reductase from cells cultured in microtiter wells: a screening assay for anticarcinogenic enzyme inducers. Anal. Biochem, 169(1988)328-336.

[23] W.H. Habig M.J. Pabst, W.B. Jakoby, Glutathione S-transferase, the first enzymic step in mercapturic acid formation, J. Biol. Chem. 249(1974)7130-7139. 
[24] T.P.H. Akerboom, H. Sies, Assay of glutathione, glutathione disulfide, and glutathione mixed disulfides in biological samples, Methods Enzymol. 7(1981)373382.

[25] M.M. Bradford, A rapid and sensitive method for the quantitation of microgram quantities of protein utilising the principle of protein-dye binding, Anal. Biochem. 72(1976)248-254.

[26] A.G. Schwartz, G.M. Prysak, C.H. Bock, M.L. Cote, The molecular epidemiology of cancer, Carcinogenesis 28(2007)507-518.

C. Ioannides, D.F.V. Lewis, Cytochromes P450 in the bioactivation of chemicals. Curr. Topics Medicin. Chem. 4(2004)1767-1788.

[28] K.L. Wall, W. Gao, J.M. TeKoppele, G.Y. Kwei, F.C. Kauffman, R.G. Thurman, The liver plays a central role in the mechanism of chemical carcinogenesis due to polycyclic aromatic hydrocarbons, Carcinogenesis 12(1991)783-786.

[29] K.R. Kaderlik, R.F. Minchin G.J. Mulder, K.F. Ilett, M. Daugaard-Jenson, C.H. Teitel, F.F. Kadlubar, Metabolic activation pathway for the formation of DNA adducts of the carcinogen 2-amino-1-methyl-6-phenylimidazo[4,5-b]pyridine (PhIP) in rat extrahepatic tissues, Carcinogenesis 15(1994)1703-1709.

[30] K. Alexandrov, M. Rojas, O. Geneste, M. Castegnaro, A-M. Camus, S. Petruzzelli, C. Giuntini, H. Bartsch, An improved fluorometric assay for dosimetry of benzo(a)pyrene diol-epoxide-DNA adducts in smokers' lung - comparisons with total bulky adducts and aryl-hydrocarbon hydroxylase-activity. Cancer Res. 52(1992)62486253.

[31] H. Bartsch, M. Castegnaro, M, Rojas, A.-M. Camus, K. Alexandrov, M. Lang, Expression of pulmonary cytochrome P4501A1 and carcinogen adduct formation in high risk subjects for tobacco-related lung cancer, Toxicol. Lett. 64/65(1992)477-483. 
[32] S. Anttila, H. Vainio, H. Hietanen, A.-M. Camus, C. Malaveille, G. Brun, K. Husgafvel-Pursiainen, L. Heikkila, A. Karjalainen, H. Bartsch, Immunohistochemical detection of pulmonary cytochrome P450IA and metabolic activities associated with P450IA1 and P450IA2 isozymes in lung cancer patients, Envtl. Hlth Persp. 98(1992)179-182.

[33] N. Hanlon, A. Okpara, N. Coldham, M.J. Sauer, C. Ioannides, Modulation of rat hepatic and pulmonary cytochromes P450 and Phase II enzyme systems by erucin, an isothiocyanate structurally related to sulforaphane, J. Agric. Food Chem. (2008) In Press.

[34] M.-S. Lee, Enzyme induction and comparative oxidative desulfuration of isothiocyanates to isocyanates. Chem. Res. Toxicol,. 9(1996)1072-1078.

[35] H.M. Bolt, P.H. Roos, in: C. Ioannides, (Ed.), Cytochromes P450: Role in the Metabolism and Toxicity of Drugs and other Xenobiotics, RSC Publishing, Cambridge, 2008 pp. 46-94.

[36] N. Hanlon, C.L. Poynton, N. Coldham, M.J. Sauer, C. Ioannides, The aliphatic isothiocyanates erucin and sulforaphane do not effectively up-regulate quinone reductase in human liver compared with rat, Submitted.

[37] P.J. Sherratt, J.D. Hayes, Glutathione $S$-transferases, in: C. Ioannides, (Ed.), Enzyme Systems that Metabolise Drugs and other Xenobiotics, John Wiley \& Sons, Ltd., Chichester, 2002 pp. 319-352.

[38] N. Hanlon, N. Coldham, A. Gielbert, N. Kuhnert, M.J. Sauer, L.J. King, C. Ioannides, Absolute bioavailability and dose-dependent pharmacokinetic behaviour of dietary doses of the chemopreventive isothiocyanate sulforaphane in the rat, $\mathrm{Br}$. J. Nut. 99(2008)559-564. 
[39] A.A. Al Janobi, R.F. Mithen, A.V. Gasper, P.N. Shaw, R.J. Middleton, C.A. Ortori, D.A. Barrett, Quantitative measurement of sulforaphane, iberin and their mercapturic acid pathway metabolites in human plasma and urine using liquid chromatography-tandem electrospray ionisation mass spectrometry, J. Chromatogr. B. 844(2006)223-234.

[40] A. Seow, H. Vainio, M.C. Yu, Effect of glutathione-S-transferase polymorphisms on the cancer preventive potential of isothiocyanates: An epidemiological perspective, Mut. Res. 592(2005)58-67.

[41] R.M. Bheemreddy, E.H. Jeffery, The metabolic fate of purified glucoraphanin in F344 rats, J. Agric. Food Chem. 55(2007)2861-2866.

[42] K. Kassahun, M. Davis, P. Hu, B. Martin, T. Baillie, Biotransformation of the naturally occurring isothiocyanate sulforaphane in the rat: Identification of phase I metabolites and glutathione conjugates, Chem. Res. Toxicol. 10(1997)1228-1233. 


\section{Figure legends}

Figure 1: Effect of erucin on cytochrome P450, phase II enzyme activities and glutathione concentrations in rat lung slices.

Lung slices were incubated in modified RPMI 1640 culture medium containing erucin ( 0 to $50 \mu \mathrm{M})$ for $24 \mathrm{~h}$. Values represent the mean $\pm \mathrm{SD}$ where $\mathrm{n}=3$, each replicate comprising up to 12 slices. EROD, ethoxyresorufin $O$-deethylase; PROD, pentoxyresorufin $O$-depentylase; QR, quinone reductase; GST, glutathione $S$ transferase. $*, \mathrm{P}<0.05, * *, \mathrm{P}<0.01$.

Figure 2: Effect of sulforaphane on cytochrome P450, phase II enzyme activities and glutathione concentrations in rat lung slices.

Lung slices were incubated in modified RPMI 1640 culture medium containing sulforaphane ( 0 to $50 \mu \mathrm{M})$ for $24 \mathrm{~h}$. Values represent the mean \pm SD where $n=3$, each replicate comprising up to 12 slices. EROD, ethoxyresorufin $O$-deethylase; PROD, pentoxyresorufin $O$-depentylase; QR, quinone reductase; GST, glutathione $S$ transferase. ${ }^{*}, \mathrm{P}<0.05, * *, \mathrm{P}<0.01$. 
Figure 3: Modulation by erucin (A) and sulforaphane (B) of pulmonary cytochrome $\mathbf{P 4 5 0}$ protein expression.

Lung slices were incubated in modified RPMI 1640 culture medium containing either erucin or sulforaphane (0 to $50 \mu \mathrm{M})$ for $24 \mathrm{~h}$. Pulmonary microsomal proteins were resolved by $10 \%(\mathrm{w} / \mathrm{v})$ SDS-PAGE and then transferred electrophoretically to Hybond-P polyvinylidene difluoride membranes. Immunoblot analysis was carried out using rat antibodies to cytochrome P450 proteins, followed by the appropriate peroxidase-labelled secondary antibody. Each lane was loaded with 10 (CYP2B1, and CYP3A2) or 40 (CYP1A and CYP1B1) $\mu \mathrm{g}$ of total protein. Molecular markers were run concurrently to aid band identification. The values in italics show optical density percentage of each band relative to control

Figure 4: Modulation by erucin (A) and sulforaphane (B) of pulmonary phase II enzyme protein expression.

Lung slices were incubated in modified RPMI 1640 culture medium containing either erucin or sulforaphane (0 to $50 \mu \mathrm{M})$ for $24 \mathrm{~h}$. Pulmonary microsomal proteins were resolved by $10 \%(\mathrm{w} / \mathrm{v})$ SDS-PAGE and then transferred electrophoretically to Hybond-P polyvinylidene difluoride membranes. Immunoblot analysis was carried out using rat antibodies to phase II enzymes, followed by the appropriate peroxidaselabelled secondary antibody. Each lane was loaded with 10 (GST $\pi$ ) or 20 (GSTa, $\mathrm{GST} \mu$ and $\mathrm{QR}) \mu \mathrm{g}$ of total protein. Molecular markers were run concurrently to aid band identification. The values in italics show optical density percentage of each band relative to control. QR, quinone reductase; GST, glutathione $S$-transferase.

Figure 5: Lactate dehydrogenase (LDH) leakage from rat lung slices incubated with erucin or sulforaphane. 
LDH leakage was determined following 24-h incubation of lung slices in modified RPMI 1640 containing a range of concentrations ( 0 to $100 \mu \mathrm{M})$ of either erucin or sulforaphane. LDH release is expressed as percentage of total LDH, i.e. the sum of the concentration retained in the slice plus the concentration leaked. Values are presented as mean $\pm \mathrm{SD}$ of duplicate measurements from each of three slices per concentration. *, $\mathrm{P}<0.05, * *, \mathrm{P}<0.01$ and $* * *, \mathrm{P}<0.001$. 